\title{
Peertechz
}

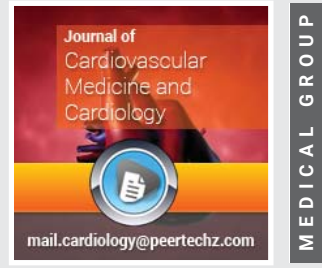

\section{A rare case of concomitant acute occlusion of left main coronary artery and right coronary artery with characteristic electrocardiographic pattern}

\section{Thilina Jayasekara ${ }^{1 *}$, Hansa Sooriyagoda' ${ }^{1}$ Dayananda}

Balasooriya', Indika Wickramatunga', Lakshman Bandara', Arulkumar Jegavanthan', Ghanamoorthi Mayurathan², Subhashini Jayawickreme ${ }^{2}$, Ajith Kularatne ${ }^{2}$ and Thilakshi

\section{Abeysinghe $^{3}$}

${ }^{1}$ Senior Registrar in Cardiology, National Hospital Kandy, Sri Lanka

${ }^{2}$ Consultant Cardiologist, National Hospital Kandy, Sri Lanka

${ }^{3}$ Research Assistant, National Hospital Kandy, Sri Lanka

\begin{abstract}
Acute Left Main Coronary Artery (LMCA) occlusion is a rare clinical presentation which often manifests as a cardiogenic shock with worse prognosis. However, the clinical outcome depends on the age of the patient, co-morbidities, the patency and dominancy of Right coronary artery. Since LMCA supplies a large myocardial territory of left ventricle, it shows a characteristic Electrocardiographic (ECG) pattern which helps to an early diagnosis. Presence of ST elevation in aVR with ST depressions of more than six leads is highly characteristic for LMCA occlusion. Here we are reporting an extremely rare case of acute concomitant occlusion of LMCA and Right coronary artery manifesting as a cardiogenic shock with ST elevation in aVR ,V1,III, aVF leads with ST depressions in all other leads. Patient showed excellent clinical outcome and reversal of characteristic ECG pattern following Percutaneous Coronary Intervention (PCI) to the culprit vessels.
\end{abstract}

\section{Introduction}

Twelve Lead ECG plays a key role in diagnosis of acute myocardial infarction and localizing the culprit vessel which enables revascularization of correct vessels in emergency setting. Acute Left main Coronary Artery (LMCA) occlusion is a devastating clinical entity with poor prognosis due to high risk of developing cardiogenic shock and refractory arrhythmias. The clinical outcome depends on the age of the patients, presence of cardiogenic shock with pulmonary oedema and the presence of dominant Right Coronary Artery (RCA) [1]. Acute Left Main occlusion manifests as characteristic ECG change with ST elevations in aVR, V1 leads and ST depressions in more
Received: 03 February, 2021

Accepted: 13 February, 2021

Published: 15 February, 2021

*Corresponding author: Thilina Jayasekara, Senior Registrar in Cardiology, National Hospital Kandy, Sri Lanka, Tel: +94777739969;

E-mail: thilinajayasekara02@gmail.com

ORCID: https://orcid.org/0000-0002-8866-3559

Keywords: Left main coronary artery occlusion; Electrocardiography; Percutaneous coronary intervention https://www.peertechz.com

Check for updates than six leads [2]. However, ECG manifestation of concomitant occlusion of LMCA and RCA is not well described in current literature.

In our clinical vignette, the presence of characteristic ECG changes of LMCA involvement with ST elevation in aVR, V1,III,aVF and ST depressions in all other leads were an entirely puzzling experience till the diagnosis of concomitant LMCA and RCA involvement was confirmed by coronary angiogram. In addition to the rarity of peculiar ECG changes, this clinical case also enlightens the challenges that had been encountered by the interventionist in catheterization laboratory in terms of selecting appropriate revascularization strategy in the emergency setting. 


\section{Although Coronary Artery Bypass Graft (CABG) is} recommended for haemodynamically stable patients with LMCA involvement the optimal revascularization strategy in concomitant acute LMCA and RCA occlusion in haemodynamically unstable patients is a grey area in coronary interventional arena. However, considering the limiting time factor with necessity of urgent revascularization Percutaenous Coronary Intervention (PCI) was selected over the CABG which is having its own limitations in acute settings.

\section{Case report}

A 48 year old Sri Lankan lady with a history of dyslipidemia and recent onset of angina presented to the local hospital with ischemic chest pain and autonomic symptoms. On admission her PR 113 min, BP 80/60mmHg with elevated jugular venous pressure and bibasal crepitations suggestive of acute heart failure. The rest of the examination was normal.

On emergency department ECG revealed ST elevation in aVR, V1, III, aVF and ST depressions in all other leads (Figure 1), provisional diagnosis of acute left main occlusion was made.

She was urgently loaded with Aspirin 300mg, Clopidogrel 300mg, Atrovastatin 80mg and Tenecteplase was given considering the time taken to transfer to a Primary PCI centre. Despite thrombolysis therapy she was having persistent angina with low blood pressure. Intravenous Dobutamin $20 \mu \mathrm{g} / \mathrm{kg} / \mathrm{min}$ and intravenous Noradrenaline $0.6 \mathrm{mg} / \mathrm{kg} / \mathrm{min}$ were started and she was transferred to the tertiary care centre for specialized management. At the tertiary care centre she was found to have ejection fraction of $40 \%$ with global hypokinesia and underwent emergency coronary angiogram via radial access. The coronary angiogram revealed $99 \%$ stenosis of osteal LMCA, $80 \%$ stenosis of Proximal Left Anterior Descending Artery (LAD), 90\% stenosis of Mid Left Circumflex Artery (LCX) with acute total occlusion of proximal Right Coronary Artery (RCA) (Figure 2a,2b). Further her Troponin I titre was $101.08 \mathrm{ng} / \mathrm{ml}$ $(<0.03 \mathrm{ng} / \mathrm{ml})$ and other investigations were normal.

Considering necessity of urgent revascularization, PCI was selected over the emergency $C A B G$ as the mode of revascularization strategy after loading with remaining dose of Clopidogrel $300 \mathrm{mg}$. RCA was initially revascularized with two 3X33 mm Drug Eluting Stents (DES) before dealing with left coronary system. Once Thrombolysis in Myocardial Infarction (TIMI) III blood flow was established in RCA, mid LCX lesion was stented with $2.75 \mathrm{X} 18 \mathrm{~mm}$ DES. As the final step of the procedure, $3.5 \mathrm{X} 33 \mathrm{~mm}$ DES was deployed from left main osteum to proximal LAD (Figure 3a,3b).

After full revascularization, ECG findings completely reversed (Figure 4). Subsequently she was managed in the coronary care unit and antiplatelet regime was changed into daily dose of Aspirin 150mg and twice a daily dose of Ticagrelor $90 \mathrm{mg}$ with other medications. After second day of CCU care she was completely free from ionotropic requirement and her ejection fraction improved up to $50 \%$ on the fourth day at the time of discharge.

\section{Discussion}

Electrocardiography (ECG) is a widely available cheap mode of diagnostic modality of Acute Myocardial Infarction (AMI) and it also helps to localize the culprit vessels. The characteristic ECG findings may further facilitate the revascurlization strategy in emergency settings in terms of selecting appropriate instrumentation for relevant coronary arterial territory in catheterization laboratory.

Since acute LMCA occlusion results in poor clinical outcome, it is of a paramount importance to have an early diagnosis with available resources. Though aVR lead is one of the neglected leads in twelve lead ECG, the presence of ST elevation in aVR with ST depressions in other leads are highly predictive of LMCA or triple vessel disease [2]. Further, ST elevation in aVR and anterior leads with wide spread ST depressions in other leads may indicate LMCA occlusion [3]. However, as in our case ST elevation in aVR , V1, III and aVF with ST depressions in all other leads were not well described as the concomitant LMCA and RCA involvement in current literature. The diagnosis was completely mystified till the diagnostic coronary angiogram was performed.

According to the study conducted by Yamuji, et al. [4] lead aVR ST segment elevation (>0.05mv) than V1 lead indicates acute LMCA occlusion with poor clinical outcome than LAD occlusion. However it is not specific to the LMCA occlusion

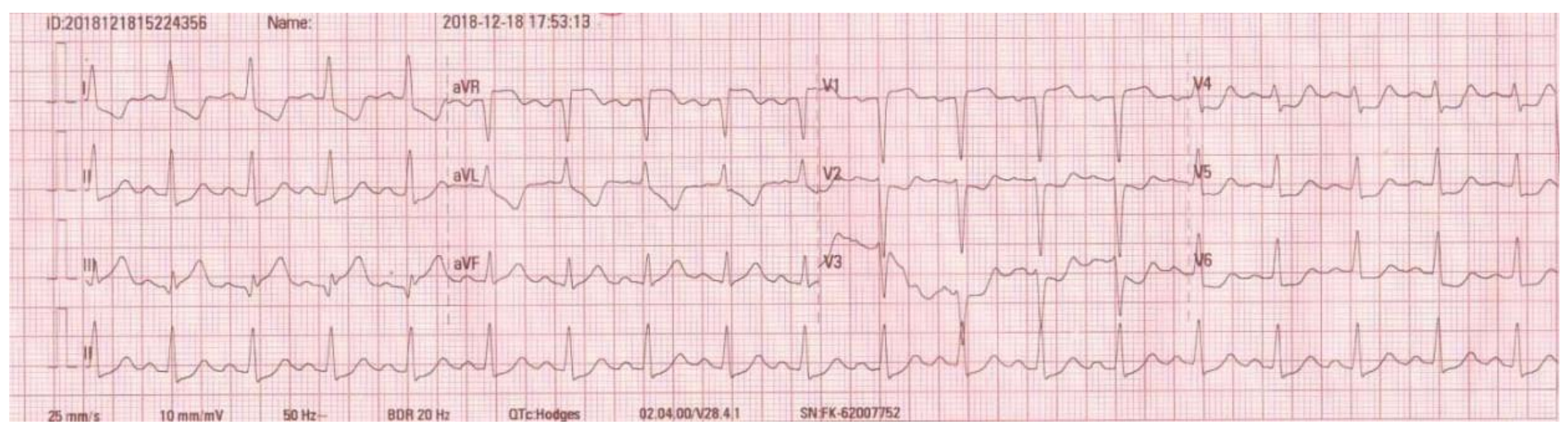

Citation: Jayasekara T, Sooriyagoda H, Balasooriya D, Wickramatunga I, Bandara L, et al. (2021) A rare case of concomitant acute occlusion of left main coronary artery and right coronary artery with characteristic electrocardiographic pattern. J Cardiovasc Med Cardiol 8(1): 010-013. 
which was further emphasized by Engelen, et al. [5] as aVR ST elevation was observed in proximal LAD occlusion prior to the first major septal branch.

Acute occlusion of LMCA accounts for poor clinical outcome due to lethal arrhythmias and cardiogenic shock. However, the presence of coexisting acute occlusion of RCA may further deteriorate already existing devastating clinical outcome. According to the available literature acute occlusion of LMCA accounts for $0.8 \%$ of primary PCI [6]. Despite lacking of clear guidance for optimal reperfusion strategies in acute LMCA occlusion sensible evidence based approach is essential for optimal clinical outcome.

Presence of critical haemodynamically instability of these patients precludes undergoing CABG in emergency setting. Further PCI provides rapid reperfusion of infarct related
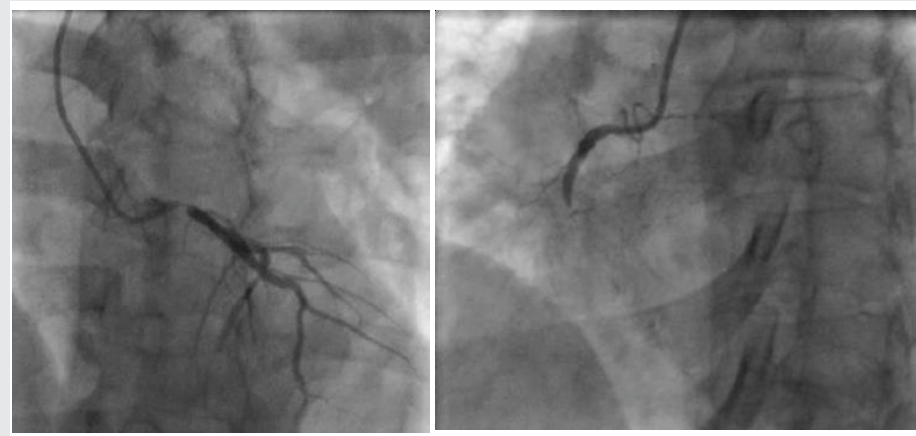

Figure 2a,b: Coronary Angiogram before procedure.

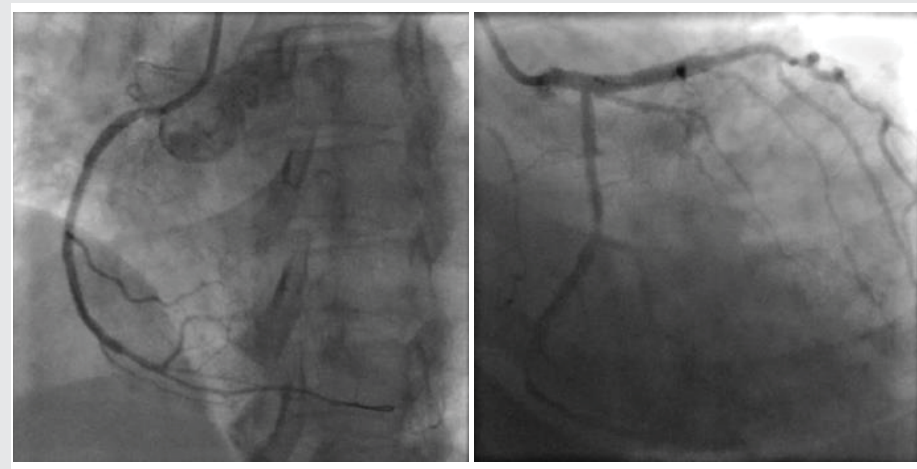

Figure 3a,b: Coronary Angiogram after procedure. artery and promptly reverse the haemodynamic instability and lethal arrhythmias. According to the expert consensus, PCI is advocated over the CABG in the context of acute LMCA occlusion for haemodynamically unstable patients with less than Thrombolysis in Myocardial Infarction (TIMI) flow grade 3, cardiogenic shock with life threatening arrhythmias and the presence of coexisting diseases that further aggravate the CABG related complications (Ex: COPD) [7].

In addition to the above benefits patients who had undergone PCI show less risk for stroke in comparison to the CABG (03\% vs. 207\%, p= 0.009) [8]. This is mainly because the fact that $C A B G$ patients are exposed to more aortic manipulation and cannulation than the patients with PCI. Considering our patient, presence of additional acute occlusion of dominant RCA may further deteriorate the clinical stability which intern warrants prompt reperfusion strategy with available resources.

Once PCI was selected over the emergency CABG, our main challenge was to deal with two culprit vessels. So reperfusion of RCA was selected to provide more haemodynamic support prior to engage with LMCA. Once the TIMI 3 flow was established in the RCA territory, non infarct related LCX was stented to optimize haemodynamic support further. These initial measures helped to deal with LMCA intervention more confidently as the final step of the procedure with an excellent clinical outcome.

Further, improvement of ejection fraction on discharge enlightens the dynamic nature of cardiac function in acute myocardial ischemia and the importance of prompt revascularization to salvage the ischemic myocardium. The latter had been performed in a timely fashion in our clinical case.

\section{Conclusion}

In this rare clinical case we try to emphasize the importance of 12 lead ECG with very peculiar ECG findings which help to diagnose the rare entity of angiographically proven concomitant acute occlusion of LMCA and RCA. It also further highlights the effectiveness of primary PCI over the emergency CABG as the rapid mode of reperfusion strategy in this extremely rare clinical presentation.

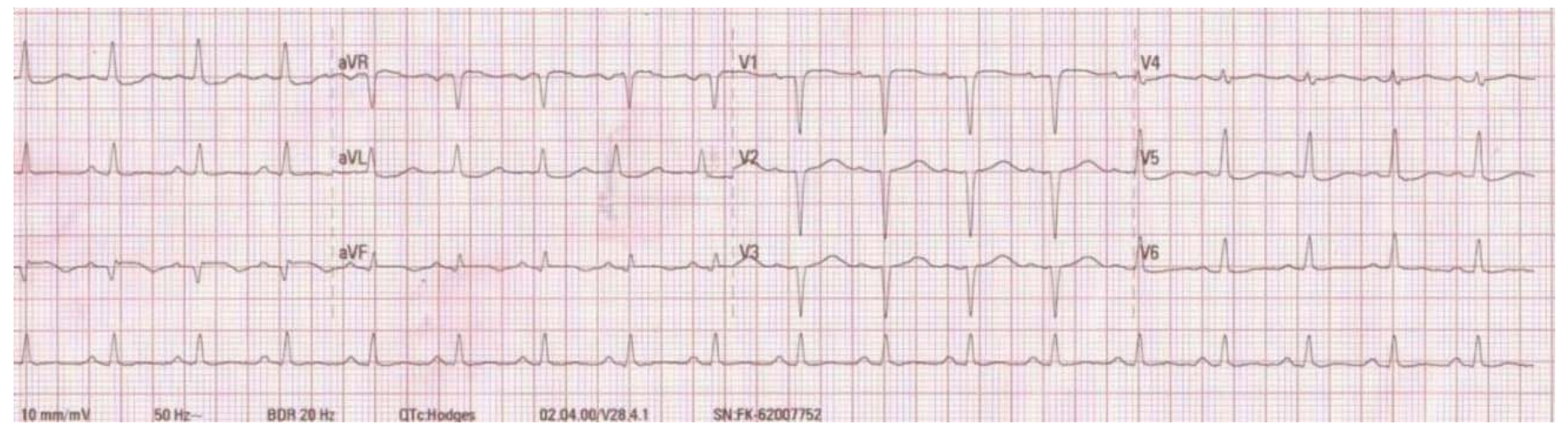




\section{References}

1. Quigley RL, Milano CA, Smith LR, White WD, Rankin JS, et al. (1993) Prognosis and management of anterolateral myocardial infarction in patients with severe left main disease and cardiogenic shock. The left main shock syndrome. Circulation 88: II65-70. Link: https://bit.ly/3u2F8eW

2. Gorgels APM, Engelen DJM, Wellens HJJ (2001) Lead aVR, a mostly ignored but very valuable lead in clinical electrocardiography. J Am Coll Cardiol 38: 1355-1356. Link: https://bit.ly/3dfydZQ

3. Nikus KC, Eskola MJ (2008) Electrocardiogram patterns in acute left main coronary artery occlusion. J Electrocardiol 41: 626-629. Link: https://bit.ly/3baLqR3

4. Yamaji H, Iwasaki K, Kusachi S, Murakami T, Hirami R, et al. (2001) Prediction of acute left main coronary artery obstruction by 12-lead electrocardiography. J Am Coll Cardiol 38: 1348-1354. Link: https://bit.ly/3jNS5nU

5. Engelen DJ, Gorgels AP, Cheriex EC, De Muinck ED, Ophuis AJ, et al. (1999) Value of the electrocardiogram in localizing the occlusion site in the left anterior descending coronary artery in acute anterior myocardial infarction. $J$ Am Coll Cardiol 34: 389-395. Link: https://bit.ly/3puhyEp

6. De Luca G, Suryapranata H, Thomas K, van 't Hof AW, de Boer MJ, et al. (2003) Outcome in patients treated with primary angioplasty for acute myocardial infarction due to left main coronary artery occlusion. Am J Cardiol 91: 235238. Link: https://bit.ly/3al1z7h

7. Antman EM, Anbe DT, Armstrong PW, Bates ER, Green LA, et al. (2004) ACC/AHA guidelines for the management of patients with ST-elevation myocardial infarction executive summary: a report of the American College of Cardiology/ American Heart Association Task Force on Practice Guidelines (Writing Committee to Revise the 1999 Guidelines for the Management of Patients With Acute Myocardial Infarction). Circulation 110: 588-636. Link: https://bit.ly/3dePG4k

8. Serruys PW, Morice MC, Kappentein AP, Colombo A, Holmes DR, et al. (2009) Percutaneous coronary interventions versus coronary-artery bypass grafting for severe coronary artery disease. N Engl J Med 360: 961-972. Link: https://bit.ly/37eXwXQ

\section{Discover a bigger Impact and Visibility of your article publication with} Peertechz Publications

\section{Highlights}

* Signatory publisher of ORCID

* Signatory Publisher of DORA (San Francisco Declaration on Research Assessment)

* Articles archived in worlds' renowned service providers such as Portico, CNKI, AGRIS, TDNet, Base (Bielefeld University Library), CrossRef, Scilit, J-Gate etc.

* Journals indexed in ICMJE, SHERPA/ROMEO, Google Scholar etc.

* OAI-PMH (Open Archives Initiative Protocol for Metadata Harvesting)

* Dedicated Editorial Board for every journa

* Accurate and rapid peer-review process

* Increased citations of published articles through promotions

* Reduced timeline for article publication

Submit your articles and experience a new surge in publication services (https://www.peertechz.com/submission).

Peertechz journals wishes everlasting success in your every endeavours.

Copyright: @ 2021 Jayasekara T, et al. This is an open-access article distributed under the terms of the Creative Commons Attribution License, which permits unrestricted use, distribution, and reproduction in any medium, provided the original author and source are credited.

Citation: Jayasekara T, Sooriyagoda H, Balasooriya D, Wickramatunga I, Bandara L, et al. (2021) A rare case of concomitant acute occlusion of left main coronary artery and right coronary artery with characteristic electrocardiographic pattern. J Cardiovasc Med Cardiol 8(1): 010-013. 